\title{
Etude Géotechnique Des Sols Compressibles : Caractérisation, Mécanisme Et Recommandation (Cas Des Régions De Berrechid Et Kenitra, Maroc)
}

\author{
Haytam Tribak, PhD \\ Département de l'Environnement, Faculté des Sciences et Techniques, \\ Université Sidi Mohamed Ben Abdellah, Fès, Maroc \\ Ayoub Belkacem, ING \\ Laboratoire Publique d'Essais et d'Etudes, Casablanca, Maroc \\ Abdelkader El Garouani, PES \\ Abderrahim Lahrach, PES \\ Département de l'Environnement, Faculté des Sciences et Techniques, \\ Université Sidi Mohamed Ben Abdellah, Fès, Maroc
}

Doi:10.19044/esj.2020.v16n9p321 ～URL:http://dx.doi.org/10.19044/esj.2020.v16n9p321

\section{Résumé}

Les constructions sur sols compressibles causent des dommages importants (stabilité, tassement, déformation, etc.) à court et à long terme partout dans le monde. Au Maroc, les zones dont les sols montrent une forte compressibilité correspondent le plus souvent à des plaines côtières récentes, comme celle de Berrechid et celle de Gharb. Les essais géotechniques réalisés au laboratoire permettent d'identifier et de savoir le comportement mécanique des sols étudiés. Parmi les tests effectués dans le cadre de ce travail : l'analyse granulométrique, le test par le bleu de méthylène, les essais des limites d'Atterberg et les essais œdométriques. D'après les résultats des essais d'identification, les deux sols sont de nature limono-argileuse dont la fraction argileuse correspond à une kaolinite, avec une granulométrie inférieure à 80 $\mu \mathrm{m}$, une valeur de bleu de méthylène entre 1,5 et 2,5; et un indice de plasticité (IP) d'une valeur de 7 pour le sol de Berrechid et de 24 pour le sol du Gharb. Les coefficients de compressibilité, obtenus grâce aux essais œdométriques, indiquent que les sols de Berrechid sont moyennement compressibles avec $\mathrm{Cc}$ $=0,156$, et les sols du Gharb sont assez fortement compressibles avec $\mathrm{Cc}=$ 0,252 . Le choix de solution d'amélioration des sols vient après l'interprétation des résultats des essais. Dans notre cas, le renforcement recommandé, afin de corriger les problèmes de déformation et de tassement, est de réaliser des colonnes ballastés et des inclusions rigides; tout dépend du type d'ouvrage élevé sur ces sols compressibles. 
Mots-clés : Sols Compressibles, Essais Géotechniques, Plaine De Berrechid, Plaine De Gharb

\title{
Geotechnical Study of Compressible Soils: Characterization, mechanism and recommendation (case of Berrechid and Kenitra regions, Morocco)
}

\author{
Haytam Tribak, PhD \\ Département de l'Environnement, Faculté des Sciences et Techniques, \\ Université Sidi Mohamed Ben Abdellah, Fès, Maroc \\ Ayoub Belkacem, ING \\ Laboratoire Publique d'Essais et d'Etudes, Casablanca, Maroc \\ Abdelkader El Garouani, PES \\ Abderrahim Lahrach, PES \\ Département de l'Environnement, Faculté des Sciences et Techniques, \\ Université Sidi Mohamed Ben Abdellah, Fès, Maroc
}

\begin{abstract}
Constructions on compressible soils cause significant damage (stability, compaction, deformation) in the short and long term in the world. In Morocco, the areas whose soils show high compressibility correspond most often to recent coastal plains, like that of Berrechid and that of Gharb. Geotechnical tests carried out in the laboratory allow to identify and know the mechanical behavior of the studied soils. Among the tests carried out in the context of this work: particle size analysis, the methylene blue test, the Atterberg limit tests and the oedometric tests. According to the results of identification tests, both soils are silty-clay nature, the clay fraction corresponds to a kaolinite, with a granulometry less than $80 \mu \mathrm{m}$, a methylene bleu value between 1,5 and 2,5 ; and a plasticity index (PI) of a value of 7 for Berrechid's soil and 24 for Gharb's soil. The compressibility coefficients, obtained by the odometric tests, indicate that Berrechid's soils are moderately compressible with $\mathrm{Cc}=0.156$, and the Gharb's soils are quite highly compressibles with $\mathrm{Cc}=0.252$. The choice of the soil improvement solution comes after the interpretation of the test results. In our case, the reinforcement recommended in ordre to correct the problems of deformation and
\end{abstract}


compaction, is to make ballasted columns and rigid inclusions ; it all depends on the type of structure raised on these compressible soils.

Keywords: Compressible Soils, Geotechnical Tests, Berrechid Plain, Gharb Plain

\section{Introduction}

Le terme compressible qualifie d'une manière générale tous matériaux dont le volume peut être réduit sous l'action d'une force extérieure. Un sol sera dit compressible s'il est susceptible d'affaissement ou de tassement. Le tassement d'un sol compressible n'est pas un processus instantané mais progressif au fil du temps (AQC, 2008).

La variabilité spatiale des sols est l'une des principales sources d'endommagement des ouvrages (La Broderie \& Breysse, 2007). Elle résulte des processus de dépôt naturels des sols (Bolle, 1994), ou anthropiques (Breysse, 2001).

Avant d'entreprendre la réalisation d'un projet de construction, il est indispensable de procéder à une reconnaissance du sol, afin de connaitre le mode de fondation approprié qui assurera la stabilité de l'ouvrage, tout en étant le plus économique possible. Quand il s'agit des sols compressibles de surface, l'étude géotechnique permettra de connaitre leur nature, les caractéristiques physiques, hydrauliques et mécaniques, en d'autres termes, leur constitution, leur consistance, leur résistance et leur déformabilité. Pour mieux cerner les caractéristiques des sols des zones étudiées, il est souhaitable de confronter les données géologiques avec celles des résultats des essais géotechniques.

Les sols compressibles dont les caractéristiques de résistance mécaniques sont faibles, induisent des tassements et des déformations sous l'effet des sollicitations apportées par les aménagements (remblais, fondations des ouvrages d'art,..). Dans les deux régions d'étude, les sols compressibles sont rencontrés principalement dans les vallées des oueds (Loukous, Sebou, Tahadart, N'fifikh, etc.) et dans les zones marécageuses. Ces sols sont de deux ordres :

- $\quad$ soit ils sont peu épais auquel cas leur substitution est envisageable

- $\quad$ soit ils se développent sur des épaisseurs importantes et des techniques spéciales sont alors nécessaires.

Dans les deux cas, une étude approfondie est nécessaire afin de les identifier, les analyser, et enfin de savoir la méthode convenable quant à leur traitement.

Le présent travail constitue une contribution à une étude des caractéristiques géotechniques mécaniques de deux types de sols compressibles notamment dans la région de Berrechid et du Gharb. L'objectif 
principal est de réaliser des études sur la compressibilité des sols dans deux plaines différentes, grâce aux différents essais d'identifications et mécaniques, afin de faire une comparaison des résultats, et d'envisager les meilleurs recommandations pour remédier aux différents problèmes rencontrés.

\section{Régions d'étude}

Les zones d'études concernées par ce travail ; sont des sites dans la plaine de Berrechid au sud-est de la ville de Berrechid et dans la plaine du Gharb (Région de Kenitra), comme indiqué dans la figure 1 par des carreaux roses.

La plaine de Berrechid et la plaine de Gharb sont parmi les plus grandes plaines côtières du Maroc caractérisées par la présence des sols instables de formation récente.

La plaine de Berrechid est un bassin subsident de la Meseta, où les mers secondaires et tertiaires ont apporté des dépôts plus ou moins puissants au cours de leurs transgressions successives et où se sont accumulés au cours du Quaternaire des limons argilo-sableux (Ruhard, 1973).

L'ensemble de la plaine de Berrechid se présente en surface comme une fosse de subsidence, limitée au Sud par les calcaires marneux du crétacé, et ailleurs par des formations primaires (schistes et quartzites). La quasitotalité de cette plaine (Fig. 2) est recouverte par des limons argileux rouges quaternaires (DGH, 1997).

L'évolution géologique de la plaine de Berrechid est marquée à la fin du tertiaire par les mouvements tectoniques du pliocène qui ont engendré le soulèvement du plateau de Settat et par conséquent, l'individualisation de la plaine de Berrechid (DGH, 1997). 


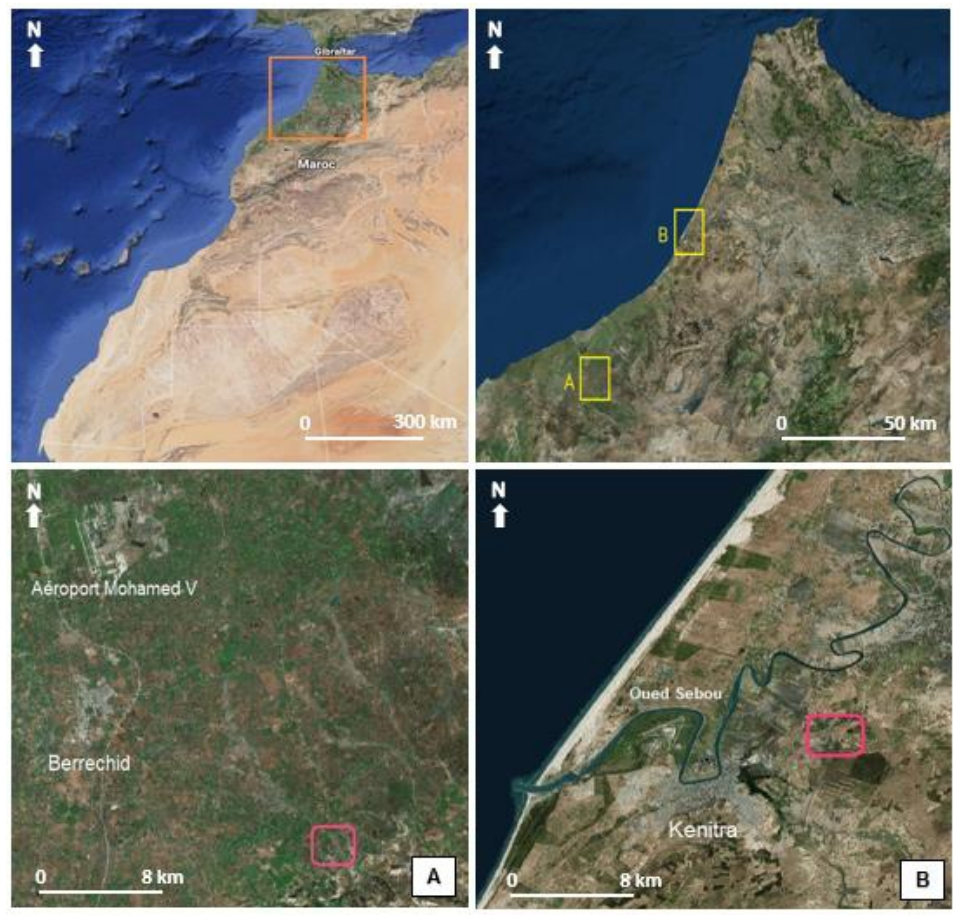

Figure 1 : Situation géographique du site d'échantillonnage sur la plaine de Berrechid (A) et sur la plaine du Gharb (B)

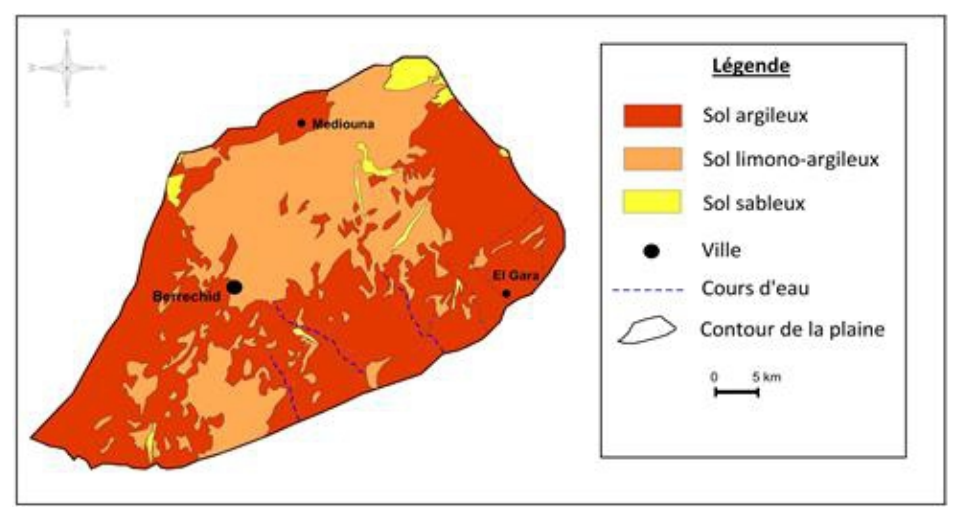

Figure 2 : Pédologie de la plaine de Berrechid (Source : Elfarrak et al., 2014, modifiée)

La plaine du Gharb s'étend sur une superficie de $4200 \mathrm{~km}^{2}$, elle comporte une bande de dunes côtières le long de la marge océanique. La morphologie de l'ensemble de la plaine correspond à une vaste cuvette dont les $4 / 5$ sont à une altitude inférieure à $20 \mathrm{~m}$, alors que les bordures présentent des reliefs très doux. Toutes les formations quaternaires plongent sous la plaine et seules les plus récentes affleurent (Fig.3 et 4) : le Soltanien de nature sableuse en bordure et le Rharbien argileux au centre (Kili et al., 2007). 


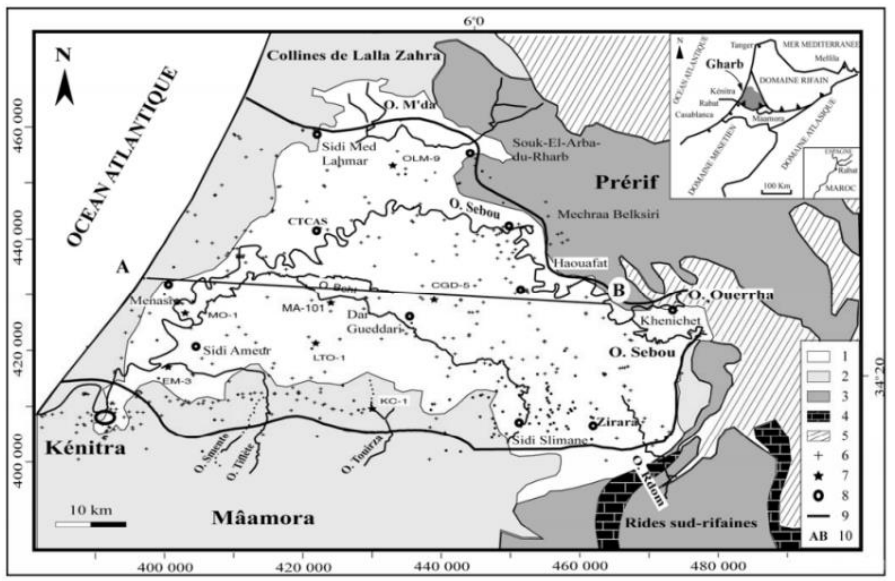

Figure 3 : Contexte géologique de la plaine de Gharb (Source : Kili et al., 2007). 1, dépôts récents ; 2, Plio-Quaternaire ; 3, Miocène (marnes) ; 4, Jurassique, (Rides sud-rifaines) ; 5 ,

Nappes prérifaines ; 6 , forages hydrogéologiques ; 7 , forages pétroliers ; 8 , stations météorologiques ; 9, limite du secteur d'étude ; 10, position de la figure 4

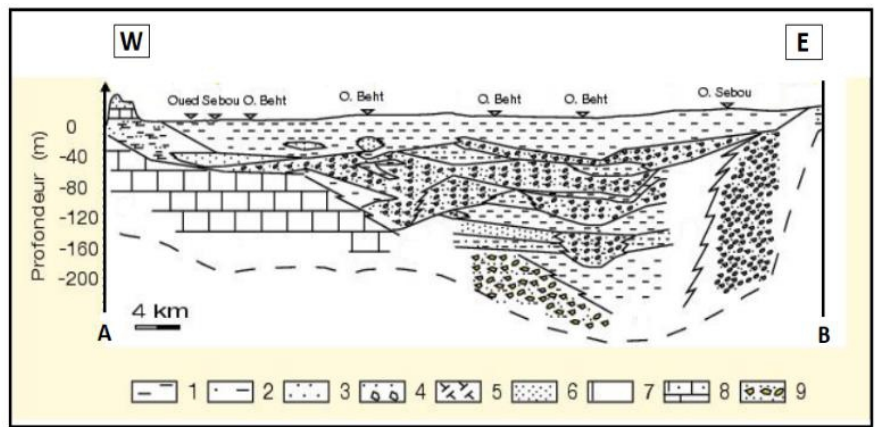

Figure 4 : Coupe géologique schématique dans la plaine de Gharb (Source : Kili et al., 2007).1. Argiles ; 2. Argiles sableuses ; 3. Sables ; 4. Sables caillouteux ; 5. Tufs :

Quaternaire moyen et supérieur continental ; 6. Sables ; 7. Grès dunaires : Quaternaire moyen et supérieur côtier ; 8. Grès calcaires ; 9. Sables et conglomérats grossiers : Pliocène et Quaternaire ancien

\section{Matériel et méthodes}

1. Matériel

Plusieurs données et outils ont été utilisés dans le travail, et qui ont aboutit à des résultats satisfaisants :

- Outils de terrain: Matériel d'échantillonnage (cylindres plastifiés normalisés, cylindres métalliques résistants, sacs plastifiés isothermes, pioche, pelle), GPS.

- Outils de laboratoire : Série de tamis, appareillages pour essais au bleu du méthylène (modèle avec burette), pénétromètre à cône et un œdomètre. 
- Données utilisées : Cartes topographiques, cartes géologiques, cartes pédologiques, articles et mémoires de thèse, différents rapports techniques, rapports et documents du Laboratoire public d'essais et d'études (LPEE) ainsi que des différents normes d'essais géotechniques (NM 13.1.008; NF P94-068; NF P94-051; NM 13.1.003).

\section{Méthodologie}

La méthodologie suivit, pour l'étude géotechnique des deux types de sols, est répartie en deux phases :

- La première consiste à une compagne d'échantillonnage de terrain. Cette dernière s'est effectuée dans la partie sud des deux plaines, dans les points GPS ( $\mathrm{X}=33,1803 / \mathrm{Y}=-7,46180$ pour la plaine de Berrechid ; $X=34,2874$ / $Y=-6,52989$ pour la plaine de Gharb), sur différents types de sol, par différentes méthodes d'extraction, de conservation et de transport des échantillons vers le laboratoire. A propos des essais d'indentifications, l'échantillonnage s'est effectué de la surface vers une profondeur de $2 \mathrm{~m}$, en récupérant le sol dans des sacs normalisés. Quatre puits, de 2,5 m de profondeur et un espacement de $20 \mathrm{~m}$, ont été réalisés dans chaque zone d'étude, d'une part pour connaitre la lithologie, et d'autre part pour l'extraction des carottes fraiches pour l'essai œdométrique. Les carottes se sont extraites de différentes profondeurs $(0,5 \mathrm{~m} ; 1 \mathrm{~m} ; 1,5 \mathrm{~m} ; 2 \mathrm{~m})$

Afin d'identifier la nature des formations et de comprendre leur comportement mécanique, une étude géotechnique détaillée a été réalisée au Laboratoire Public d'Essais et d'Etude de Casablanca (LPEE). Cette dernière, correspondant à la deuxième phase, consiste, en premier, à des essais d'identification sur les échantillons de la région de Berrechid (E1) et de la région de Kenitra (E2); l'analyse granulométrique s'est effectuée selon la norme NM 13.1.008. Les essais au bleu du méthylène et les limites d'Atterberg ont été réalisés respectivement selon les normes NF P94-068 et NF P94-051. L'essai de compressibilité à l'œdomètre vient après l'identification des sols, et est réalisé selon la norme marocaine NM 13.1.003.

\section{Résultats et discussions \\ IV. 1. Essais d'identification}

\section{IV.1.1 Analyse granulométrique}

Les résultats de l'analyse granulométrique sont résumés dans le tableau et les courbes ci-dessous $(\mathrm{E} 1=$ échantillon de Berrechid $)(\mathrm{E} 2=$ échantillon de Kenitra). Puisque les matériaux concernés sont des sols, on procède à un calcul cumulé des tamisats : 
E1

\begin{tabular}{|c|c|}
\hline Masse en l'état de la prise d'essai $(\mathrm{g})$ & $\mathrm{M}_{1}=4226$ \\
\hline Etuvage $(24 \mathrm{~h})$ & $105^{\circ} \mathrm{c}$ \\
\hline Masse sèche après étuvage $(\mathrm{g})$ & $\mathrm{M}_{2}=3600$ \\
\hline Masse sèche après lavage sur $80 \mu \mathrm{m}(\mathrm{g})$ & $\mathrm{M}_{3}=1071$ \\
\hline
\end{tabular}

\begin{tabular}{|c|c|}
\hline Masse en l'état de la prise d'essai $(\mathrm{g})$ & $\mathrm{M}_{1}=1000$ \\
\hline Etuvage $(24 \mathrm{~h})$ & $105^{\circ} \mathrm{c}$ \\
\hline Masse sèche après étuvage $(\mathrm{g})$ & $\mathrm{M}_{2}=919$ \\
\hline Masse sèche après lavage sur $80 \mu \mathrm{m}(\mathrm{g})$ & $\mathrm{M}_{3}=308$ \\
\hline
\end{tabular}

Tableau 1 : Résultats de l'analyse granulométrique des échantillons E1 et E2

\begin{tabular}{|c|c|c|c|c|c|c|}
\hline \multirow[t]{2}{*}{$\begin{array}{l}\text { Maille carrée } \\
\quad(\mathbf{m m})\end{array}$} & \multicolumn{2}{|c|}{$\begin{array}{c}\text { Refus cumulés } \mathbf{R} \\
\text { (g) }\end{array}$} & \multicolumn{2}{|c|}{$\begin{array}{l}\text { Refus cumulés } \mathbf{r} \\
(\%)\end{array}$} & \multicolumn{2}{|c|}{ Tamisats (\%) } \\
\hline & E1 & E2 & E1 & E2 & E1 & E2 \\
\hline 16.000 & 3.0 & - & - & - & 100 & 100 \\
\hline 12.500 & 16,4 & - & - & - & 100 & 100 \\
\hline 10.000 & 23,7 & - & 1 & - & 99 & 100 \\
\hline 8.000 & 32,5 & - & 1 & - & 99 & 100 \\
\hline 6.300 & 41,1 & - & 1 & - & 99 & 100 \\
\hline 5.000 & 53,4 & - & 1 & - & 98,5 & 100 \\
\hline 4.000 & 71,5 & - & 2 & - & 98 & 100 \\
\hline 3.150 & 91,1 & - & 3 & - & 97 & 100 \\
\hline 2.500 & 124,3 & - & 3 & - & 97 & 100 \\
\hline 2.000 & 167,6 & - & 5 & - & 95 & 100 \\
\hline 1.600 & 210,4 & - & 6 & - & 94 & 100 \\
\hline 1.250 & 261,8 & - & 7 & - & 93 & 100 \\
\hline 1.000 & 305,7 & 2,3 & 8 & - & 92 & 100 \\
\hline 0.800 & 354,8 & 3,8 & 10 & - & 90 & 100 \\
\hline 0.630 & 402,9 & 5,3 & 11 & 1 & 89 & 99 \\
\hline 0.500 & 459,2 & 7,1 & 13 & 1 & 87 & 99 \\
\hline 0.400 & 514,8 & 9,6 & 14 & 1 & 86 & 99 \\
\hline 0.315 & 582,9 & 18,5 & 16 & 2 & 84 & 98 \\
\hline 0.250 & 625,4 & 38,3 & 17 & 4 & 83 & 96 \\
\hline 0.200 & 751,7 & 131,2 & 21 & 14 & 79 & 86 \\
\hline 0.160 & 885,5 & 217,0 & 25 & 24 & 75 & 76 \\
\hline 0.100 & 1027,1 & 300,3 & 29 & 33 & 71 & 67 \\
\hline 0.080 & 1061,0 & 306,7 & 29.5 & 33.4 & 70.5 & 66.6 \\
\hline
\end{tabular}




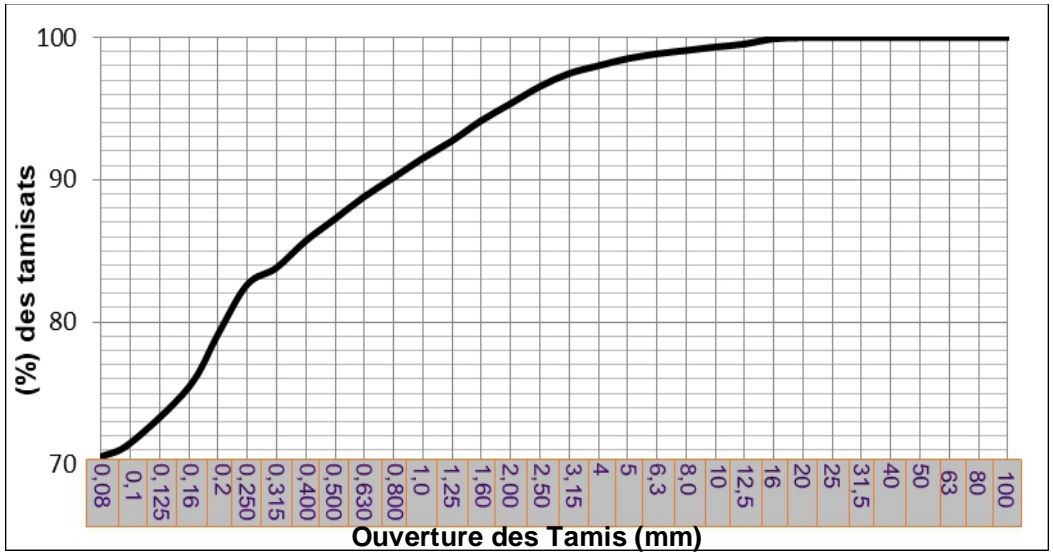

Figure 5 : courbe d'analyse granulométrique du sol de la plaine de Berrechid (E1)

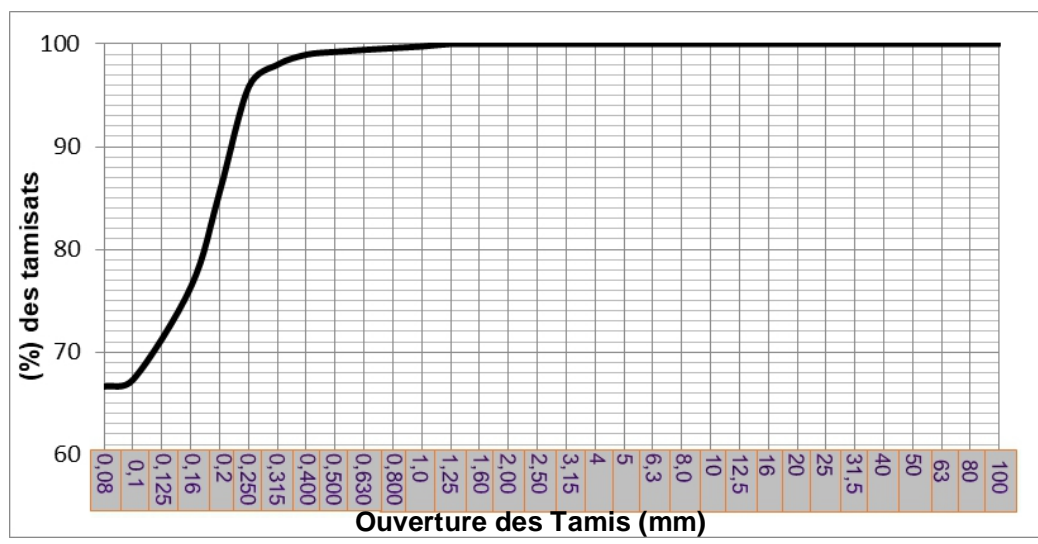

Figure 6 : courbe d'analyse granulométrique du sol de la plaine de Gharb (E2)

A partir du tableau et des courbes, l'échantillon E2 présente une granulométrie plus fine. Nous pouvons déduire d'avance que les sols du Gharb seront plus compressibles que ceux de Berrechid.

\section{IV.1.2 Détermination de la valeur de bleu du méthylène}

Les argiles sont des matériaux ayant plusieurs caractéristiques physiques, notamment connues pour leur capacité d'absorption. Cependant certaines argiles n'absorbent pas les solutions chimiques, d'où l'essai au bleu de méthylène est indispensable en géotechnique pour déterminer la propreté des matériaux par rapport à leur constitution argileuse.

Pour l'échantillon E1, la valeur du bleu de méthylène pour le sol est :

$\mathrm{VBS}=\mathbf{2 , 1 0 9} \mathrm{g}$ de bleu de méthylène pour $100 \mathrm{~g}$ de sol sec.

Pour l'échantillon E2, la valeur du bleu de méthylène pour le sol est :

VBS = 1,76 $\mathrm{g}$ de bleu de méthylène pour $100 \mathrm{~g}$ de sol sec. 
D'après le guide des terrassements routiers (GTR 92) : les deux échantillons correspondent à des sols fins de nature limono-argileuse par ce que la valeur de VBS est comprise entre 1,5 et 2,5. La partie argileuse correspond à la Kaolinite.

\section{IV.1.3 Détermination des limites d'Atterberg}

La limite de liquidité équivaut, à la teneur en eau d'un sol au point de passage entre l'état liquide et l'état plastique.

La limite de plasticité est la teneur en eau d'un sol remanié au point de transition entre les états plastique et solide.

L'objectif de l'essai est de déterminer l'indice de plasticité (I.P) qui correspond à la différence entre la limite de liquidité et la limite de plasticité, cet indice définit l'étendu du domaine plastique.

Tableau 2 : Résultats des limites d'Atterberg des échantillons E1 et E2

\begin{tabular}{|c|c|c|c|c|c|}
\hline \multicolumn{2}{|c|}{$\begin{array}{c}\text { Limite de liquidité WL } \\
(\%)\end{array}$} & \multicolumn{2}{|c|}{$\begin{array}{c}\text { Limite de plasticité WP } \\
(\%)\end{array}$} & \multicolumn{2}{|c|}{ Indice de plasticité (IP) } \\
\hline E1 & E2 & E1 & E2 & E1 & E2 \\
\hline 26 & 40 & 19 & 16 & 7 & 24 \\
\hline
\end{tabular}

D'après la norme française NF P 94-051, le sol E1 est classé moyennement plastique, tandis que le sol E2 est classé plastique.

\section{IV.2. Essais de compressibilité par palier à l'œdomètre}

L'essai à l'œdomètre a pour but de caractériser le comportement des sols de type argileux et permet ainsi de définir, en complément des limites d'Atterberg, la possibilité de gonflement-retrait du matériau. L'essai s'applique à tous les types de sols, naturels ou reconstitués au laboratoire, utilisés dans le domaine de la construction. Il permet de déterminer les caractéristiques suivantes : Le coefficient de compressibilité $\mathrm{C}_{\mathrm{c}}$, le coefficient de gonflement $\mathrm{C}_{\mathrm{g}}$, la pression de pré-consolidation $\boldsymbol{\sigma}_{\mathrm{c}}$, la pression de gonflement $\boldsymbol{\sigma}_{\mathrm{g}}$ et le coefficient de consolidation verticale $\mathrm{C}_{\mathrm{v}}$.

Les courbes de compressibilité œdométriques suivantes sont la représentation, en échelle semi-logarithmique des indices des vides (à la stabilisation) en fonction des contraintes, des échantillons E1 et E2 (Fig. 5 et 6) : 


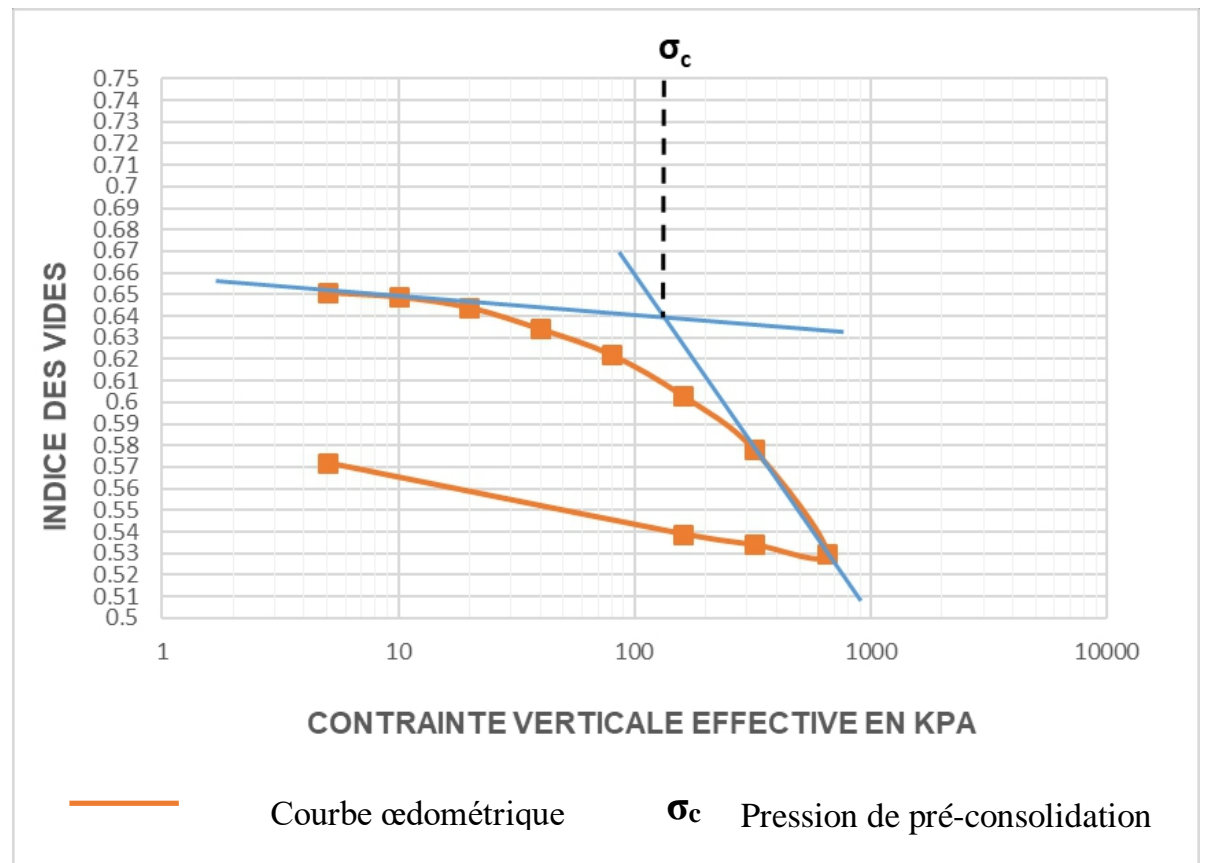

Figure 7 : Courbe de compressibilité œdométrique pour l'échantillon E1

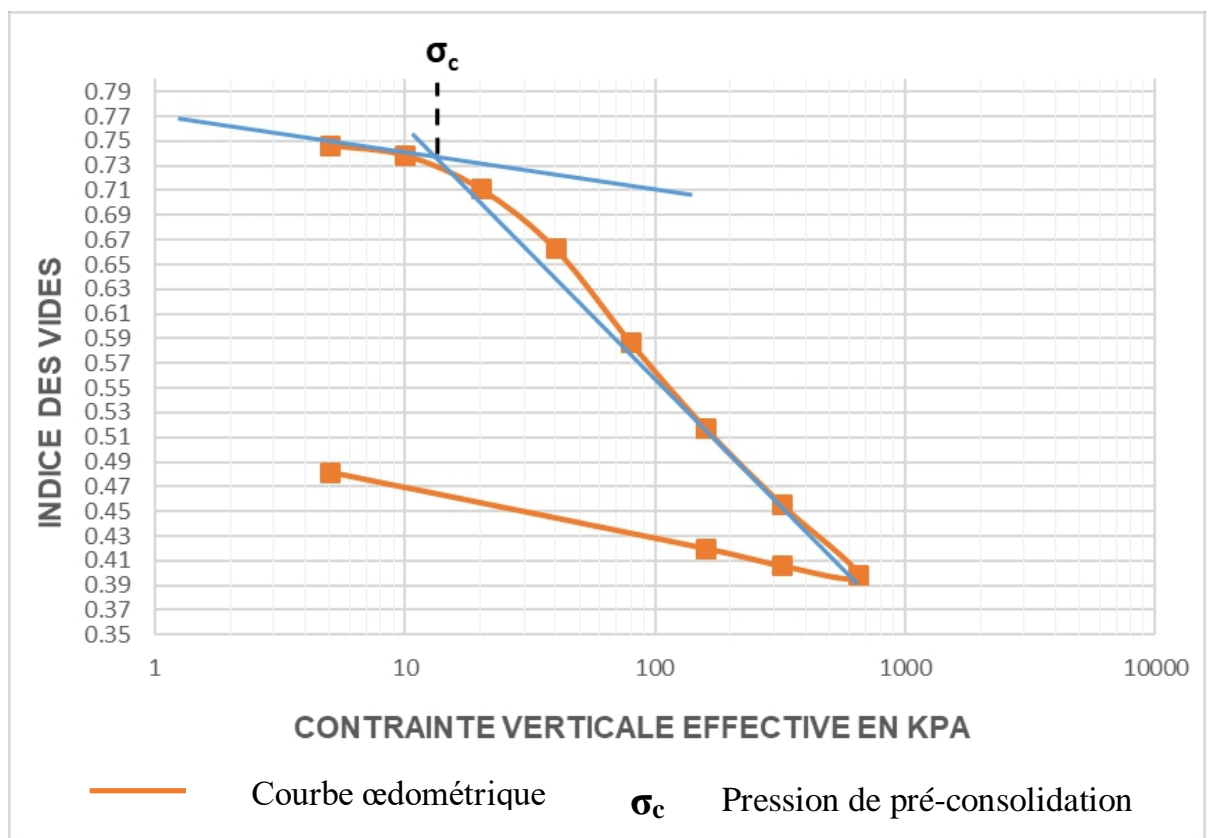

Figure 8 : Courbe de compressibilité œdométrique pour l'échantillon E2

A partir de la courbe on détermine les caractéristiques suivantes :

- Le coefficient de compressibilité $\mathbf{C}_{\boldsymbol{c}}$ : qui est la pente de la droite qui ajuste les derniers points de la partie inclinée de la courbe. 
$\mathbf{C}_{\mathbf{c}}(\mathbf{E 1})=\mathbf{0 , 1 5 6} ;$ d'après la norme il s'agit d'un sol moyennement $\mathbf{C}_{\mathbf{c}}(\mathbf{E 2})=\mathbf{0 , 2 5 2}$; d'après la norme il s'agit d'un sol assez fortement compressible

- Le coefficient de gonflement $\mathbf{C}_{\mathbf{g}}$ : est la pente de la droite qui ajuste les derniers points de la partie de déchargement.\#

$\mathbf{C}_{\mathrm{g}}(\mathbf{E 1})=\mathbf{0 , 0 2 1}<\mathbf{0 , 0 4}$ seuil des matériaux gonflants

$\mathbf{C}_{\mathrm{g}}(\mathbf{E 2})=\mathbf{0 , 0 4 1}>\mathbf{0 , 0 4}$ seuil des matériaux gonflants

Les courbes de consolidation verticale $(\mathrm{Cv})$ de CASAGRANDE suivantes sont la représentation des tassements de l'éprouvette en fonction du temps, pour une contrainte de $320 \mathrm{kPa}$ :

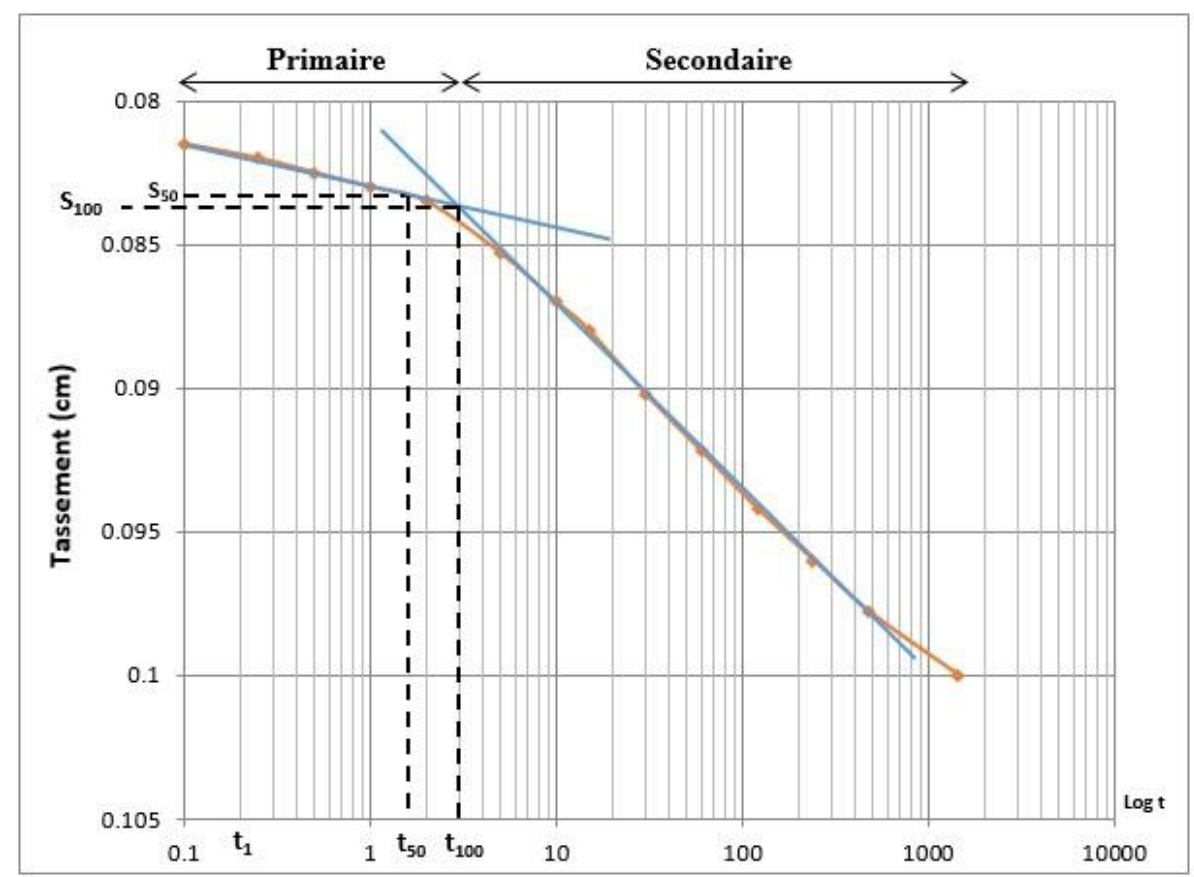

Figure 9 : Courbe de consolidation œdométrique pour une contrainte de $320 \mathrm{kPa}$ pour l'échantillon E1

$$
\mathrm{C}_{\mathrm{v}}(\mathrm{E} 1)=\mathbf{2}, \mathbf{1 7} \times \mathbf{1 0}^{-7} \mathrm{~m}^{2} / \mathrm{s}
$$




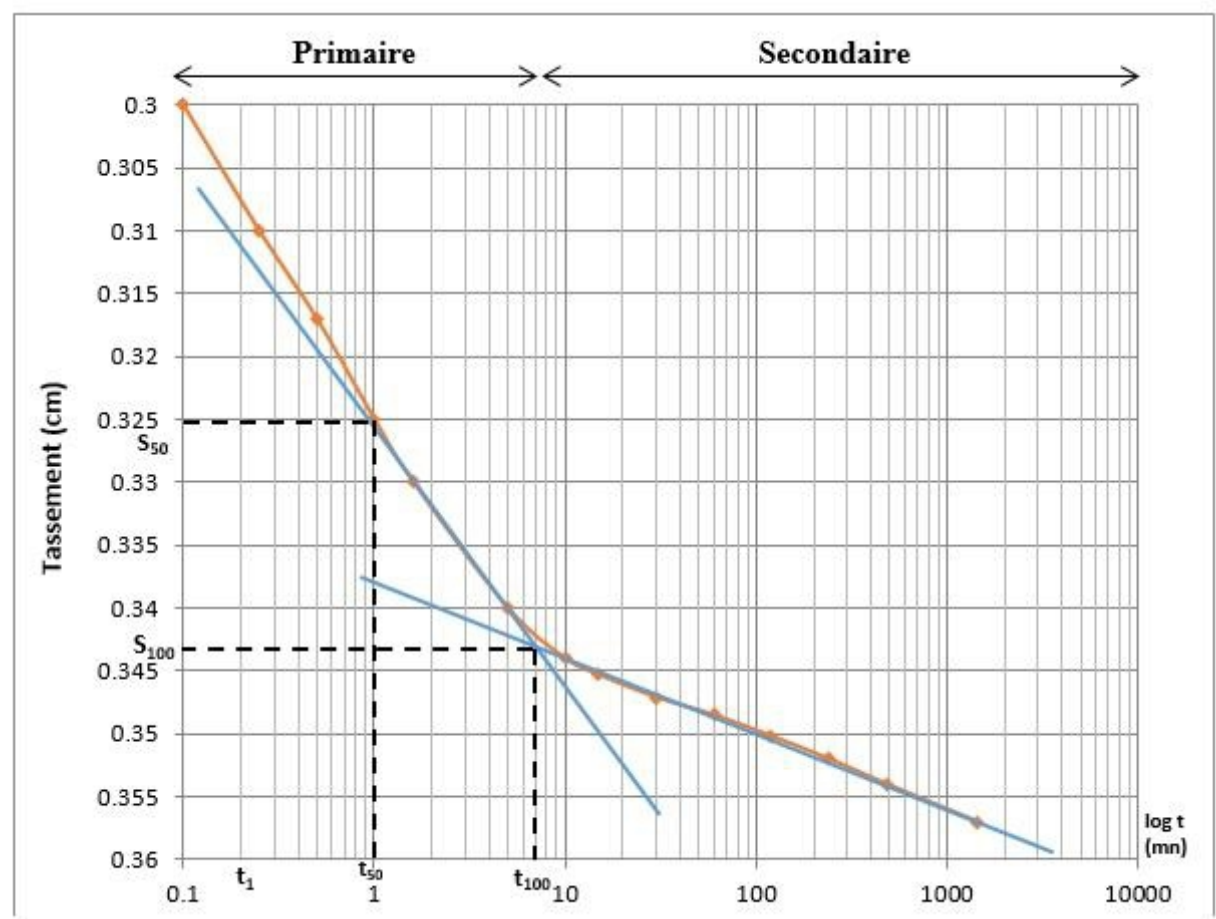

Figure 10 : Courbe de consolidation œdométrique pour une contrainte de $320 \mathrm{kPa}$ pour l'échantillon E2

$$
\mathrm{C}_{\mathrm{v}}(\mathrm{E} 2)=\mathbf{3 , 6 2} \times \mathbf{1 0}^{-7} \mathrm{~m}^{2} / \mathrm{s}
$$

La partie argileuse des deux sols correspond à une kaolinite, par ce que $\mathrm{Cv}$ est comprise entre $2.10^{-7}$ et $\mathbf{4 . 1 0 ^ { - 7 }}$.

En comparant les deux plaines, les sols de la plaine de Gharb présentent une compressibilité plus élevée que celle de la plaine de Berrechid, en raison de la légère différence de la nature et de la granulométrie des grains. Généralement, ces sols auront besoin d'une amélioration et d'un renforcement s'ils joueront le rôle d'un support pour des prochaines constructions.

L'essai œdométrique est l'essai mécanique le plus important. En effet, c'est grâce à lui qu'on peut savoir si le sol présente une compressibilité ou non, ainsi que le degré de son tassement, ensuite la détermination du choix idéal du type de renforcement. Les sols du Gharb ont montré des compressibilités plus élevées que ceux de Berrechid.

L'élévation de tous types de constructions (remblais, ouvrages, fondations, etc.) sur des sols compressibles pose de nombreux problèmes associés à la faible résistance de ces sols et à leur forte compressibilité. Lorsque le profil théorique de la construction est stable et le tassement du sol de fondation est acceptable, aucun traitement particulier n'est à appliquer. 
Dans le cas contraire, on peut procéder à l'amélioration du sol de fondation ou au renforcement du la construction elle-même (KHEMISSA, 1999).

Le renforcement des sols est un domaine récent et particulier pour leur l'amélioration. Il recouvre des techniques qui consistent à placer des inclusions résistantes au sein du sol (SCHLOSSER et al., 1984).

A l'heure actuelle, les problèmes de tassement rencontrés dans les deux plaines concernent en grande partie les remblais destinés à un usage routier; ceci est en rapport principalement avec la longueur des trajets traversant ces sols compressibles et des incertitudes lors des calculs des résultats. A titre d'exemple, la dégradation de la chaussée de la route provinciale $\mathrm{N}^{\circ} 3613$, reliant la ville de Berrechid à Ouled $\mathrm{S}$ 'ïd, construite en 2005 sur des sols compressibles sur un trajet de $23 \mathrm{~km}$ a été affectée un an plus tard par plusieurs affaissements du sol.

Les techniques d'amélioration recommandées pour les sols étudiés, sont les méthodes de renforcement par des colonnes ballastées et par des inclusions rigides utilisées spécifiquement pour les sols compressibles. Les deux techniques de traitement choisies, nécessitent un budget élevé pour leur réalisation, en revanche, leur mise en œuvre est rapide et présente de bons résultats. En effet, le Maroc a connu et continu de connaitre un développement infrastructurel important ; l'ouest du Maroc, où se situent les régions d'études, est le plus concernée par ce développement. Des voies routières (autoroute Casablanca-Béni Mellal, Casablanca-Tanger, etc.), des voies ferroviaires (Ligne grande vitesse Tanger-Casablanca, Ligne grande vitesse CasablancaMarrakech...), des ouvrages d'art et d'autres types de construction sont déjà achevés, en cours d'exécution ou en phase de planification. Cependant, la nécessité du franchissement de ces zones à risque, impose, à ces différentes élévations, la problématique traitée dans le présent article. Donc, afin d'éviter tout problème de tassement et d'endommagement, surtout des infrastructures routières, ferroviaires et des ouvrages d'art, le renforcement des sols compressibles dans les deux plaines est indispensable.

\section{Conclusion}

La plaine de Berrechid et la plaine de Gharb sont parmi les plus grandes plaines côtières du Maroc qui se caractérisent par la présence des sols instables. Ces derniers correspondent à des dépôts récents (Quaternaire) qui couvrent la quasi-totalité des deux plaines.

Pour les deux régions, les essais d'identifications ont montré des caractéristiques plus ou moins semblables pour les deux types de sols :

- Au niveau de la texture, la taille de la majorité des constituants est inférieure à $80 \mu \mathrm{m}$, avec une dominance limoneuse ; ce sont donc des sols limoneux-argileux ; 
- Les valeurs de bleu de méthylène comprises entre 1.5 et 2.5 , implique que la partie argileuse correspond à la kaolinite ;

- D'après la valeur de l'indice de plasticité (I.P), le sol de la région de Berrechid est moyennement plastique ; par contre, pour celui de la région de Kenitra est plastique.

Les essais œdométriques ont dévoilé le mécanisme de la compressibilité des deux échantillons du sol étudié. A partir des résultats des coefficients de compressibilité générés par les courbes de compressibilité œdométrique, les sols de Gharb ont montré une compressibilité assez forte, quant aux sols de Berrechid ont montré une compressibilité moyenne. Grâce aux deux courbes de consolidation verticale, nous avons pu déduire le temps des trois phases de tassement (immédiat, primaire et secondaire), ainsi qu'approuver, une fois de plus, la nature de la fraction argileuse contenue dans les deux sols qui correspond à la kaolinite et cela à partir des résultats de coefficient de consolidation.

Les différents essais et analyses réalisés en laboratoire sur ces types de sols ont montré des résultats inquiétants quant à leur stabilité ; ceci nécessite de trouver des solutions convenables d'amélioration et de renforcement, lors de la construction des remblais et des ouvrages.

Parmi les techniques de renforcement recommandées, les colonnes ballastées et les inclusions rigides qui ont prouvé leur efficacité afin de résoudre les problèmes de tassement et d'instabilité des sols.

\section{References:}

1. AQC (AGENCE QUALITE CONSTRUCTION). (2008). Mouvements de fondations de maisons individuelles. Première partie : Tassements courants. https://fr.scribd.com/document/53933557/Tassement-courantfichea01, (consulté le : 17/02/2020).

2. BOLLE A. (1994). How to manage the spatial variability of natural soils, probabilities and materials, ed. D. Breysse, Kluwer, 505-516.

3. BREYSSE D. (2001). Les enjeux de la modélisation des terrains et des structures pour décrire au mieux l'interaction sol-ouvrage, Proc. Of A. Caquot Conference, ed. Presses ENPC, Paris.

4. DGH (DIRECTION GENERALE DE L'HYDRAULIQUE). (1997). Etude de la plaine de Berrechid, mission 1. Description et analyse. Edition définitive (Annexes A et B).

5. ELFARRAK H., HAKDAOUI M. \& FIKRI A. (2014). Development of Vulnerability through the DRASTIC Method and Geographic Information System (GIS) (Case Groundwater of 
Berrchid), Morocco. Journal of Geographic Information System, Vol 6, $\mathrm{N}^{\mathrm{o}} 1,14 \mathrm{p}$.

6. KHEMISSA M. (1999). Méthodologie d'étude et règles de constructions des remblais sur sols compressibles, actes de séminaire sur les terrassements dans les grands travaux, ARAL, At Sétif, Algérie.

7. KILI M., EL MANSOURI B., TAKY A. \& CHAO J. (2007). Nouvelle approche d'estimation des prélèvements d'eau d'irrigation à partir des ressources souterraines, Rabat. Section Sciences de la Terre, 2006, ${ }^{\circ} 28,31-39$.

8. LA BORDERIE C, ET BREYSSE D. (2007). Étude du tassement différentiel sur sols hétérogènes, Revue européenne de génie civile 11(4), $453-462$.

9. NF 94-051. (1993). Sols: Reconnaissances et Essais. Détermination des limites d'Atterberg-limite de liquidité à la coupelle - limite de plasticité au Rouleau. ISSN 0335-3931, AFNOR.

10. NF 94-068. (1998). Sols : Reconnaissances et Essais. Mesure de la capacité d'adsorption de bleu de méthylène d'un sol ou d'un matériaux rocheux par l'essai à la tache - ISSN 0335-3931, AFNOR.

11. NM 13.1.003. (1998). Essai de compressibilité par paliers à l'œdomètre. SNIMA.

12. NM 13.1.008. (1999). Analyse granulométrique des sols par tamisage. SNIMA.

13. RUHARD J.P. (1973). Plaine de Berrechid et basse-Chaouia entre Casablanca et Mohammedia, Ressources en eau du Maroc « Tome $2 », 185 \mathrm{p}$.

14. Schlosser F., Jacobsen H.M. \& Juran I. (1984). Renforcement des sols. Revue Française de Géotechnique, N 29, 7 - 33. 